\title{
The clinical benefit of implementing guidelines in cardiovascular disease prevention in real world settings
}

\author{
Vasilios G. Athyros ${ }^{1}$, Thomas D. Gossios², Niki Katsiki ${ }^{3}$, Asterios Karagiannis ${ }^{1}$, Dimitri P. Mikhailidis ${ }^{3}$
}

\begin{abstract}
12nd Propedeutic Department of Internal Medicine, Medical School, Aristotle University of Thessaloniki, Hippocration Hospital, Thessaloniki, Greece

${ }^{2} 1^{\text {st }}$ Cardiology Clinic, Medical School, Aristotle University of Thessaloniki, AHEPA Hospital, Thessaloniki, Greece

${ }^{3}$ Department of Clinical Biochemistry (Vascular Disease Prevention Clinic) and Department of Surgery, Royal Free Campus, University College London Medical School, University College London (UCL), London, United Kingdom
\end{abstract}

Submitted: 4 February 2012

Accepted: 4 February 2012

Arch Med Sci 2012; 8, 1: 6-10

DOI: 10.5114/aoms.2012.27272

Copyright (C) 2012 Termedia \& Banach

In the current issue of the Archives of Medical Science two studies from the same group, an academic community cardiology practice [1, 2], address the issue of the impact of the implementation of secondary cardiovascular disease (CVD) prevention guidelines on the long-term clinical outcome in patients with established coronary artery disease (CAD).

In the $1^{\text {st }}$ study [1] the differences in medication usage and in CVD event rates are reported. From the early era (before 2002) to the later era (20052008 ), there was an increase in the use of $\beta$-blockers (from $66 \%$ to $83 \%$, $p<0.0001$ ), angiotensin-converting enzyme inhibitors (ACE-I) or angiotensin receptor blockers (ARBs) (from $34 \%$ to $80 \%, p<0.0001$ ), and statins (from $40 \%$ to $90 \%, p<0.0001)$. This resulted in a substantial CAD rate reduction ( $9.2 \%$ vs. $29.1 \%$ in the later vs. early time period; $p<0.0001)$ [1]. In the second study that included a different population [2], CAD patients were not initially treated with statins during the first year of being seen in an outpatient cardiology practice but subsequently treated with statins $(100 \%)$ for a mean period of 66 months. Myocardial infarction (MI) occurred in $10 \%$ of patients before statins, and in $4 \%$ after statins $(p<0.01)$, percutaneous coronary intervention $(\mathrm{PCl})$ had been performed in $22 \%$ of patients before statins and in $13 \%$ after statins $(p<0.01)$, and coronary artery bypass graft (CABG) surgery had been performed in $18 \%$ of patients before statins and in $7 \%$ after statins $(p<0.001)$ [2]. These two studies point out that the implementation of secondary CVD prevention guidelines in a "real world" setting have a considerable positive impact on subsequent CVD morbidity and mortality $[1,2]$.

Several studies have demonstrated improved clinical outcomes when key quality-of-care indicators are implemented in the management of patients with acute coronary syndromes (ACS) or stable CAD [3]. However, secondary prevention guidelines can be poorly implemented [4, 5]. Particularly, in regard to statins, which have to be taken indefinitely, there is a concern that poor compliance may compromise their benefit [4-7]. Patients with dyslipidaemia do not experience symptoms and they need motivation to adhere to their medication. Clinical trials are performed at a controlled environment and data reported by them may vary from "real
Corresponding author: Dimitri P. Mikhailidis MD, FFPM, FRCP, FRCPath Department of Clinical Biochemistry (Vascular Disease Prevention Clinic) Royal Free Hospital Campus University College London Medical School University College London (UCL) Pond Street, London NW3 2QG, United Kingdom Phone: +44 2078302258 Fax: +44 2078302235 E-mail: mikhailidis@aol.com 
life data" $[6,7]$. For example a US study that reported the 2-year adherence of a non-selected MEDICAID cohort showed that only $<40 \%$ of patients were on a statin $>80 \%$ of the time [8]. The large US registry CRUSADE (Can Rapid Risk Stratification of Unstable Angina Patients Suppress Adverse Outcomes With Early Implementation of the ACC/AHA Guidelines), the EUROASPIRE I, II, III real world data on effective treatment of stable CAD, and the international Global Registry of Acute Coronary Events (GRACE) study demonstrated a world-wide underuse of proven medical therapies, including statins, among patients with either ACS or stable CAD $[4,9,10]$. Therefore, the gap between guidelines and routine clinical practice seems to have persisted during the decade 2000 to 2010 [11] and is a universal phenomenon [12-18]. Bridging the care gap in secondary CVD prevention remains a significant challenge. The lost benefit due to undertreatment contributes to the CVD burden. In addition to diet, exercise and lifestyle interventions new strategies are urgently needed to optimize vascular disease management in secondary prevention.

There have been prospective attempts to improve this situation, but mainly focused on the time after the acute event. A simplified treatment algorithm that initiated secondary CVD protection measures before hospital discharge in patients with an ACS was tested in the Cardiac Hospitalization Atherosclerosis Management Program (CHAMP) during the 90's [19]. CHAMP was associated with a significant increase in use of medications that had been demonstrated to reduce mortality after ACS. Comparison of the pre- and post-CHAMP patient groups showed that aspirin use at discharge improved from $68 \%$ to $92 \%$ ( $p<0.01$ ), $\beta$-blocker use from $12 \%$ to $62 \%$ ( $p<0.01)$, ACE-I use increased from $6 \%$ to $58 \%(p<0.01)$, and statin use increased from $6 \%$ to $86 \%(p<0.01)$. This increased use of treatment persisted during subsequent follow-up. During this programme a high percentage of patients achieved secondary CVD prevention targets including low density lipoprotein cholesterol (LDL-C) goal (< $100 \mathrm{mg} / \mathrm{dl})(58 \%$ in post-CHAMP vs. $6 \%$ pre-CHAMP, $p<0.001)$. This translated in an improvement in clinical outcome; the incidence of non-fatal MI and cardiac death was cut by half in the 1-year follow-up [19]. Another major attempt to improve implementation of guidelines was the realtime American College of Cardiology Guidelines Applied in Practice (GAP) programme. The GAP tools also resulted in higher discharge rates of secondary prevention medication (aspirin, $\beta$-blockers, ACE I and statins) [20]. The GAP tools were associated with fewer rehospitalizations for CAD, MI, and combined death/cerebrovascular event/MI in the first 1-year of follow-up. The CVD mortality substantial- ly declined during the post-GAP period [20]. The Swedish Quality Control Programme [21] is the first attempt to assess implementation of CVD treatment guidelines on a national level. After 1 year a large proportion of patients were still on preventive drugs: aspirin (96\%), $\beta$-blockers (78\%) and lipidlowering drugs (83\%), but no actual clinical results have been reported yet [21].

There have been only a few attempts to improve adherence in secondary CVD prevention drugs away from the acute event, taking into account "real word data". This is why the 2 studies $[1,2]$ published in this issue are valuable, despite their retrospective design.

From 1998 to 2002 the prospective, randomised, and controlled GReek Atorvastatin on Coronaryheart-disease Evaluation (GRACE) study was carried out [22]. This was the first target based study, aiming to assess the clinical benefit from attaining the LDL-C goal (<100 mg/dl) in 1,600 consecutive patients with established CAD. Patients were randomised either to "structured care" $(n=800)$ with dose titrating of atorvastatin (from 10 to $80 \mathrm{mg}$ / day) in order to achieve the LDL-C target or to "usual" medical care $(n=800)$. All patients were followed for a mean period of 3 years [22]. There was an orchestrated effort to keep the "structured care" patients on atorvastatin and the LDL-C $<100$ $\mathrm{mg} / \mathrm{dl}$ (mean dosage of atorvastatin: $24 \mathrm{mg} /$ day). This was achieved in $95 \%$ of patients, at a level much higher than "usual care" (12 on statins, 3\% on LDL-C target). Other secondary CVD prevention therapies had no difference between the 2 treatment groups [22]. During the study 196 (24.5\%) CAD patients on "usual" care had a CAD recurrent event or died vs. 96 (12\%) CAD patients on "structured care"; risk ratio (RR) 0.49 , confidence interval $(\mathrm{Cl})$ 0.27-0.73, $p<0.0001$. In detail, structured care reduced, in comparison to "usual care", total mortality (RR 0.57, 95\% Cl 0.39-0.78, $p=0.002$ ), coronary mortality ( $R R=.53,95 \% \mathrm{Cl} 0.29-0.74, p=0.0016)$, coronary morbidity (RR 0.46, $95 \% \mathrm{Cl} 0.25$ $0.71, p<0.0001)$, and stroke (RR $0.53,95 \% \mathrm{Cl} 0.30$ $0.82, p=0.0018$ ) [22]. All subgroups of patients (women, those with diabetes mellitus, arterial hypertension, age 60 to 75 years, congestive heart failure or prior revascularization) benefited from treatment with atorvastatin. Withdrawal of patients because of side effects from the atorvastatin group was low $(0.75 \%)$, similar to that of the "usual" care group (0.4\%) [22]. Similar were the results of the Aggressive Lipid-Lowering Initiation Abates New Cardiac Events (ALLIANCE) study, published 2 years later [23]. In ALLIANCE an aggressive, focused statin therapy management strategy outperformed "usual care" in health maintenance organization and Veterans Administration clinic patients with CAD. The "structured care" patients experienced a pri- 
mary outcome (time to first CVD event: $\mathrm{HR}, 0.83$; $95 \% \mathrm{Cl} 0.71$ to $0.97, p=0.02$ ). This reduction in morbidity was largely due to fewer non-fatal myocardial infarctions ( $47 \%$ reduction, $p=0.0002$ ) [23]. This benefit was less than that reported by GREACE, but this study came 2 years later (secondary prevention improves with time) and was performed in US, where "usual care" was better than that in Greece.

Very recent data on primary CVD prevention are very encouraging. These show that first MI (within primary CVD prevention) and subsequent short term (30 day) mortality halved over the last 10-25 years in at least 3 European countries [24-26].

From 2002 to 2010 in England, the age standardised total mortality rate fell by about half, whereas the age standardised CVD event and case fatality rates both declined by about one third. This was reported from the analysis of the data that included 840,175 people of all ages who were admitted to hospital for acute MI or died suddenly from acute MI. Half of the decline in deaths from acute MI during the 2000s in England can be attributed to a decline in event rate and half to improved survival at 30 days. Both prevention of first acute MI by addressing CVD risk factors and medical treatment during the acute phase hospitalization have contributed to the decline in deaths from acute MI over the past decade [24].

In addition, over half of the recent fall in mortality from CAD in Poland can be attributed to reductions in major risk factors and about one third to evidence based medical treatments. The CAD deaths in Poland plummeted after the socioeconomic reform in 1989. From 1991 to 2005, the death rate from CAD in Poland halved in people aged 25 74 years [25]. About 54\% of the fall was attributed to changes in risk factors, mainly reductions in total cholesterol (39\%) and an increase in physical activity (10\%). These benefits were partially offset by increases in body mass index $(-4 \%)$ and diabetes $(-2 \%)$. Blood pressure fell in women, explaining about $29 \%$ of their decrease in mortality, but rose in men generating a negative influence $(-8 \%)$. About $15 \%$ of the observed decrease in mortality was attributable to reduced smoking in men but this effect was negligible in women [25].

Finally, a study that included $\approx 250,000$ Danish people and followed them up for 25 years showed that the rate of first $\mathrm{Ml}$ and subsequent short-term mortality declined by nearly half between 1984 and 2008. The reduction in mortality occurred for all patients, independent of sex and comorbidity. However, comorbidity burden was a strong prognostic factor for short and long-term mortality, while gender was not. It was estimated that half of the decline in mortality since 1980 is attributable to primary prevention of $\mathrm{MI}$ (reduction in the prevalence of major CVD risk factors such as smoking, sedentary lifestyle, and uncontrolled high blood pressure) [26]. The other half is attributable to the introduction of thrombolysis, CABG, $\mathrm{PCl}$, and antiplatelet regimens, $\beta$-blockers, ACE-I, and statins. It is relevant that the incidence of $\mathrm{MI}$ has continued to decline despite increased prevalence of obesity and diabetes [26].

Thus, the effort to address any excess CVD risk (though lower now in absolute terms than in the past) related to comorbidies has an outstanding place in primary CVD prevention and can further reduce CVD events and mortality. This was the reason that we carried out 4 "best practice" studies (aiming in attending international guidelines for CVD prevention) in order to deal with major comorbidities in a primary care setting, mainly in primary prevention patients [27-30]. The results of these studies suggest that the estimated risk for a fatal or non-fatal CVD event was nearly halved at 6 months of treatment, by multifactorial intervention in patients with at least one major CVD risk factor (arterial hypertension, dyslipidaemia, diabetes, and metabolic syndrome) [27-30]. Another best practice study showed that this was the case for long-term morbidity and mortality also in patients with metabolic syndrome [31]. Other comorbidies, increasing CVD risk, such as chronic kidney disease [32-34] and non-alcoholic fatty liver disease [35-37] should be diagnosed and treated accordingly. Also, in patients with metabolic syndrome, multifactorial intervention resulted in elimination of new cases of diabetes [38, 39], which is considered to be as harmful as pre-existing diabetes [40]. In any case statin treatment plays a great role in primary CVD prevention. A recent meta-analysis [41] showed that statins were found to be efficacious in preventing death and CVD morbidity in people at low CVD risk (without CAD or diabetes), within primary prevention. Reductions in RR were similar to those seen in patients with a history of CAD [41].

The data suggest that there is plenty of room in real world settings for a better implementation of guidelines in secondary CVD prevention (bridging the gap between guidelines and everyday clinical practice) with substantial clinical gains. Efforts like the ones in the 2 Studies in this issue of the journal $[1,2]$ are welcomed. On the other hand, effective risk factor control in primary CVD prevention leads to fewer MIs, while effective treatment measures during the acute phase of MI results in halving short- and long-term CVD mortality. However, there is still room for further improvement by addressing major comorbidities (especially the forthcoming epidemic of diabetes), which shape acute- and long-term mortality in men and women [42]. Interventions both in primary and secondary 
CVD prevention will finally lead to substantial reduction of the human cost of this disease.

\section{Acknowledgments}

This editorial was written independently; no company or institution supported it financially. No professional writer was involved. The authors have given talks, attended conferences and participated in trials and advisory boards sponsored by various pharmaceutical companies.

\section{References}

1. Mercando AD, Lai HM, Aronow WS, et al. Reduction in atherosclerotic events: a retrospective study in an outpatient cardiology practice. Arch Med Sci 2012; 8: 57-62.

2. Lai HM, Aronow WS, Mercando AD, et al. The impact of statin therapy on long-term cardiovascular outcomes in an outpatient cardiology practice. Arch Med Sci 2012; 8: 53-6.

3. Celiński R, Grzywa-Celińska A, Myśliński W, Dybała A, Mosiewicz J. Are patients undergoing treatment of hyperlipidaemia with statins the best candidates for early cardiac rehabilitation? Arch Med Sci 2011; 7: 756-9.

4. Kotseva K, Wood D, DeBacker G, DeBacquer D, Pyörälä K, Keil U; EUROASPIRE Study Group. Cardiovascular prevention guidelines in daily practice: a comparison of EUROASPIRE I, II, and III surveys in eight European countries. Lancet 2009; 373: 929-40.

5. Pearson TA, Laurora I, Chu H, Kafonek S. The lipid treatment assessment project (L-TAP): a multicenter survey to evaluate the percentages of dyslipidemic patients receiving lipid-lowering therapy and achieving low-density lipoprotein cholesterol goals. Arch Intern Med 2000; 160: 459-67.

6. Andrade SE, Walker AM, Gottlieb LK, et al. Discontinuation of antihyperlipidemic drugs: do rates reported in clinical trials reflect rates in primary care settings? N Engl J Med 1995; 332: 1125-31.

7. Lachaine J, Rinfret S, Merikle EP, Tarride JE. Persistence and adherence to cholesterol lowering agents: evidence from Regie de l'Assurance Maladie du Quebec data. Am Heart J 2006; 152: 164-9.

8. Benner JS, Glynn RJ, Mogun H, Neumann PJ, Weinstein MC, Avorn J. Long-term persistence in use of statin therapy in elderly patients. JAMA 2002; 288: 455-61.

9. Roe MT, Peterson ED, Newby LK, et al. The influence of risk status on guideline adherence for patients with nonST-segment elevation acute coronary syndromes. Am Heart J 2006; 151: 1205-13.

10. Granger CB, Steg PG, Peterson E, et al. Medication performance measures and mortality following acute coronary syndromes. Am J Med 2005; 118: 858-65.

11. Davidson $\mathrm{MH}$. Differences between clinical trial efficacy and real-world effectiveness. Am J Manag Care 2006; 12 (15 Suppl): S405-11.

12. Klingman D, Williams SA, Benner JS, Smith TW, Ahn J, O'Donnell JC. Gauging the treatment gap in dyslipidemia: findings from the 1999-2000 National Health and Nutrition Examination Survey. Am Heart J 2005; 150: 595-601.

13. Goodman SG, Langer A, Bastien NR, et al; DYSIS Canadian Investigators. Prevalence of dyslipidemia in statin-treated patients in Canada: results of the DYSlipidemia International Study (DYSIS). Can J Cardiol 2010; 26: e330-5.

14. Niu S, Zhao D, Zhu J, et al; BRIG Project. The association between socioeconomic status of high-risk patients with coronary heart disease and the treatment rates of evidence-based medicine for coronary heart disease secondary prevention in China: Results from the Bridging the Gap on CHD Secondary Prevention in China (BRIG) Project. Am Heart J 2009; 157: 709-15.e1.

15. Rapezzi C, Biagini E, Bellis P, et al; EASY Investigators. Exploring the gap between National Cholesterol Education Program guidelines and clinical practice in secondary care: results of a cross-sectional study involving over 10000 patients followed in different specialty settings across Italy. J Cardiovasc Med (Hagerstown) 2008; 9: 878-87.

16. Steinhagen-Thiessen E, Bramlage $P$, Lösch $C$, et al. Dyslipidemia in primary care: prevalence, recognition, treatment and control: data from the German Metabolic and Cardiovascular Risk Project (GEMCAS). Cardiovasc Diabetol 2008; 7: 31-9.

17. Banegas JR, Vegazo O, Serrano P, et al; HISPALIPID Study Group Investigators. The gap between dyslipidemia control perceived by physicians and objective control patterns in Spain. Atherosclerosis 2006; 188: 420-4.

18. Schwandt P, Brady AJB. Achieving lipid goals in Europe: how large is the treatment gap? Expert Rev Cardiovasc Ther 2004; 2: 431-49.

19. Fonarow GC, Gawlinski A, Moughrabi S, Tillisch JH. Improved treatment of coronary heart disease by implementation of a Cardiac Hospitalization Atherosclerosis Management Program (CHAMP). Am J Cardiol 2001; 87: 819-22.

20. Vasaiwala S, Nolan E, Ramanath VS, et al. A quality guarantee in acute coronary syndromes: the American College of Cardiology's Guidelines Applied in Practice program taken real-time. Am Heart J 2007; 153: 16-21.

21. Stagmo M, Israelsson B, Brandström H, et al. The Swedish National Programme for Quality Control of Secondary Prevention of Coronary Artery Disease: results after one year. Eur J Cardiovasc Prev Rehabil 2004; 11: 18-24.

22. Athyros VG, Papageorgiou AA, Mercouris BR, et al. Treatment with atorvastatin to the National Cholesterol Educational Program goal versus 'usual' care in secondary coronary heart disease prevention. The GREek Atorvastatin and Coronary-heart-disease Evaluation (GREACE) study. Curr Med Res Opin 2002; 18: 220-8.

23. Koren MJ, Hunninghake DB; ALLIANCE Investigators. Clinical outcomes in managed-care patients with coronary heart disease treated aggressively in lipidlowering disease management clinics: the alliance study. J Am Coll Cardiol 2004; 44: 1772-9.

24. Smolina K, Wright FL, Rayner M, Goldacre MJ. Determinants of the decline in mortality from acute myocardial infarction in England between 2002 and 2010: linked national database study. BMJ 2012; 344: d8059. doi: 10.1136/bmj.d8059.

25. Bandosz P, O'Flaherty M, Drygas W, et al. Decline in mortality from coronary heart disease in Poland after socioeconomic transformation: modelling study. BMJ 2012; 344: d8136. doi: 10.1136/bmj.d8136.

26. Schmidt M, Jacobsen JB, Lash TL, Bøtker HE, Sørensen HT. 25 year trends in first time hospitalisation for acute myocardial infarction, subsequent short and long term mortality, and the prognostic impact of sex and comorbidity: a Danish nationwide cohort study. BMJ 2012; 344: e356. doi: 10.1136/bmj.e356. 
27. Athyros VG, Hatzitolios A, Karagiannis A, et al; INDEED Collaborative Group. Initiative for a new diabetes therapeutic approach in a Mediterranean country: the INDEED study. Curr Med Res Opin 2009; 25: 1931-40.

28. Karagiannis A, Hatzitolios Al, Athyros VG, at al. Implementation of guidelines for the management of arterial hypertension. The IMPULSION study. Open Cardiovasc Med J 2009; 3: 26-34.

29. Hatzitolios Al, Athyros VG, Karagiannis A, et al; IMPROVE Collaborative Group. Implementation of strategy for the management of overt dyslipidemia: the IMPROVEdyslipidemia study. Int I Cardiol 2009; 134: 322-9.

30. Athyros VG, Karagiannis A, Hatzitolios Al, et al.; SAGE-METS collaborative group. Standardized arrangement for a guideline-driven treatment of the metabolic syndrome: the SAGE-METS study. Curr Med Res Opin 2009; 25: 971-80.

31. Athyros VG, Ganotakis E, Kolovou GD, et al; Assessing The Treatment Effect in Metabolic Syndrome Without Perceptible Diabetes (ATTEMPT) Collaborative. Assessing the treatment effect in metabolic syndrome without perceptible diabetes (ATTEMPT): a prospective-randomized study in middle aged men and women. Curr Vasc Pharmacol 2011; 9: 647-57.

32. Cicero AF, Ertek S. Preclinical and clinical evidence of nephro- and cardiovascular protective effects of glycosaminoglycans. Arch Med Sci 2010; 6: 469-77.

33. Athyros VG, Hatzitolios Al, Karagiannis A, et al. IMproving the imPlemEntation of cuRrent guidelines for the mAnagement of major coronary hearT disease rlsk factors by multifactorial interVEntion. The IMPERATIVE renal analysis. Arch Med Sci 2011; 7: 984-92.

34. Kappagoda CT, Amsterdam EA. Improving guidelines for the management of coronary heart disease risk factors. Arch Med Sci 2011; 7: 923-4.

35. Athyros VG, Giouleme O, Ganotakis ES, et al. Safety and impact on cardiovascular events of long-term multifactorial treatment in patients with metabolic syndrome and abnormal liver function tests: a post hoc analysis of the randomised ATTEMPT study. Arch Med Sci 2011; 7 796-805.

36. Vulic D, Loncar S, Krneta M, et al. Risk factor control and adherence to treatment in patients with coronary heart disease in the Republic of Srpska, Bosnia and Herzegovina in 2005-2006. Arch Med Sci 2010; 6: 270-5.

37. Athyros VG, Tziomalos K, Gossios TD, et al. Safety and efficacy of long-term statin treatment for cardiovascular events in patients with coronary heart disease and abnormal liver tests in the Greek Atorvastatin and Coronary Heart Disease Evaluation (GREACE) Study: a post-hoc analysis. Lancet 2010; 376: 1916-22.

38. Athyros VG, Elisaf MS, Alexandrides T, et al. Long-Term Impact of Multifactorial Treatment on New-Onset Diabetes and Related Cardiovascular Events in Metabolic Syndrome: A Post Hoc ATTEMPT Analysis. Angiology 2011 (in press). PMID:22007026.

39. Aronow WS. Editorial on management of diabetes mellitus with coronary artery disease. Arch Med Sci 2011; 7: 928-30.

40. Verdecchia P, Reboldi G, Angeli F, et al. Adverse prognostic significance of new diabetes in treated hypertensive subjects. Hypertension 2004; 43: 963-9.

41. Tonelli M, Lloyd A, Clement F, et al; Alberta Kidney Disease Network. Efficacy of statins for primary prevention in people at low cardiovascular risk: a metaanalysis. CMAJ 2011; 183: E1189-202.

42. Kolovou G, Marvaki A, Bilianou H. One more look at guidelines for primary and secondary prevention of cardiovascular disease in women. Arch Med Sci 2011; 7 747-55. 